\title{
Highly Pathogenic Avian Influenza A(H5N8) Virus in Wild Migratory Birds, Qinghai Lake, China
}

\author{
Mingxin Li, ${ }^{1}$ Haizhou Liu, ${ }^{1}$ Yuhai $\mathrm{Bi},{ }^{1}$ \\ Jianqing Sun, ${ }^{1}$ Gary Wong, Di Liu, Laixing Li, \\ Juxiang Liu, Quanjiao Chen, Hanzhong Wang, \\ Yubang He, Weifeng Shi, \\ George F. Gao, Jianjun Chen
}

In May 2016, a highly pathogenic avian influenza A(H5N8) virus strain caused deaths among 3 species of wild migratory birds in Qinghai Lake, China. Genetic analysis showed that the novel reassortant virus belongs to group B H5N8 viruses and that the reassortment events likely occurred in early 2016.

Since 2003, the A/Goose/Guangdong/1/96 lineage $\mathcal{N ( G s / G d - l i n e a g e )}$ of highly pathogenic avian influenza (HPAI) A(H5N1) viruses has been evolving into diverse clades and subclades (1). A novel subclade of HPAI A(H5N8), 2.3.4.4, which evolved from a clade 2.3.4 H5N1 variant, was initially isolated from domestic ducks in eastern China in 2010 (2) and caused outbreaks in domestic ducks and migratory birds in South Korea in early 2014 $(3,4)$. In late 2014, several countries in Europe and East Asia experienced an invasion of HPAI H5N8 virus (5). This HPAI H5Nx (H5N8, H5N2, and H5N1) lineage subsequently emerged in North America, causing fatalities among wild birds and outbreaks in domestic poultry (5).

Available evidence strongly suggests that the HPAI H5N8 subclade 2.3.4.4 viruses were introduced and spread across the globe by migratory birds (6-8). Currently, 2 distinct $\mathrm{H} 5 \mathrm{~N} 8$ virus groups have been identified: group A (Buan2-like) and group B (Gochang1-like) (3). Group A

Author affiliations: CAS Key Laboratory of Special Pathogens and Biosafety, Chinese Academy of Sciences, Hubei, China (M. Li,

H. Liu, J. Liu, Q. Chen, H. Wang, J. Chen); Center for Influenza Research and Early-warning (CASCIRE), Chinese Academy of Sciences, Beijing (Y. Bi, G. Wong, D. Liu, Q. Chen, G.F. Gao,

J. Chen); Key Laboratory of Pathogenic Microbiology and Immunology, Chinese Academy of Sciences, Beijing, China (Y. Bi, G. Wong, D. Liu, G.F. Gao); Shenzhen Third People's Hospital, Shenzhen, China (Y. Bi, G.F. Gao); Qinghai Lake National Nature Reserve, Qinghai, China (J. Sun, Y. He); Northwest Institute of Plateau Biology of Chinese Academy of Sciences, Xining, China (L. Li); Institute of Pathogen Biology, Taishan Medical College, Shandong, China (W. Shi)

DOI: http://dx.doi.org/10.3201/eid2304.161866
H5N8 viruses predominate and have further evolved into 3 distinct subgroups: icA1, icA2, and icA3 (6). We report the emergence of a group B H5N8 virus in Qinghai Lake, China, a key breeding and stopover site for waterfowl along the Central Asian Flyway.

\section{The Study}

On May 1, 2016, the carcass of a brown-headed gull (Larus brunnicephalus) was found on Egg Islet, a major breeding site of bar-headed geese, in Qinghai Lake. Carcasses of wild birds were recovered for 15 consecutive days, starting on May 8: 124 bar-headed geese (Anserindicus), 17 brown-headed gulls (Larus brunnicephalus), and 14 great black-headed gulls (L. ichthyaetus). As of June 4, a total of 158 birds, most of which were bar-headed geese, were found dead in Qinghai Lake, predominantly on Egg Islet (Figure 1; online Technical Appendix, https://wwwnc.cdc. gov/EID/article/23/4/16-1866-Techapp1.pdf)

In the first 9 days of the outbreak, multiple organs (brain, intestine, liver, lung, pancreas and kidney) were collected aseptically from 18 bar-headed geese, 6 brownheaded gulls, and 1 great black-headed gull. We inoculated 10-day-old chicken embryos with the homogenates of these organs for virus isolation. Almost all organs analyzed were positive for influenza virus, and we detected only H5- and N8- subtype-specific strains.

We sequenced full-length genomes and found the polybasic amino acid sequence, REKRRKR*GL in the hemagglutinin (HA) cleavage site, confirming the virus can be classified as highly pathogenic. Sequences of 48 Qinghai Lake H5N8 influenza isolates (QH-H5N8) were deposited into the GISAID database (http://www.gisaid.org) under accession nos. EPI774110-EPI774510.

Sequence comparisons showed high nucleotide identity among all 8 gene segments of the QH-H5N8 isolates ( $>99.2 \%$; data not shown), indicating that the isolated strains are descendants of a common ancestral virus. A BLAST search (https://blast.ncbi.nlm.nih.gov/) suggested that QH-H5N8 is a reassortant virus (online Technical Appendix Table 1) and that the HA, neuraminidase (NA), and nonstructural protein (NS) genes of QH-H5N8 share high nucleotide identity ( $>99.1 \%$ ) with those of the H5N8 virus that circulated among poultry in eastern China in 2014 (A/ duck/Eastern China/S1109/2014[H5N8]). The remaining

${ }^{1}$ These authors contributed equally to this article. 

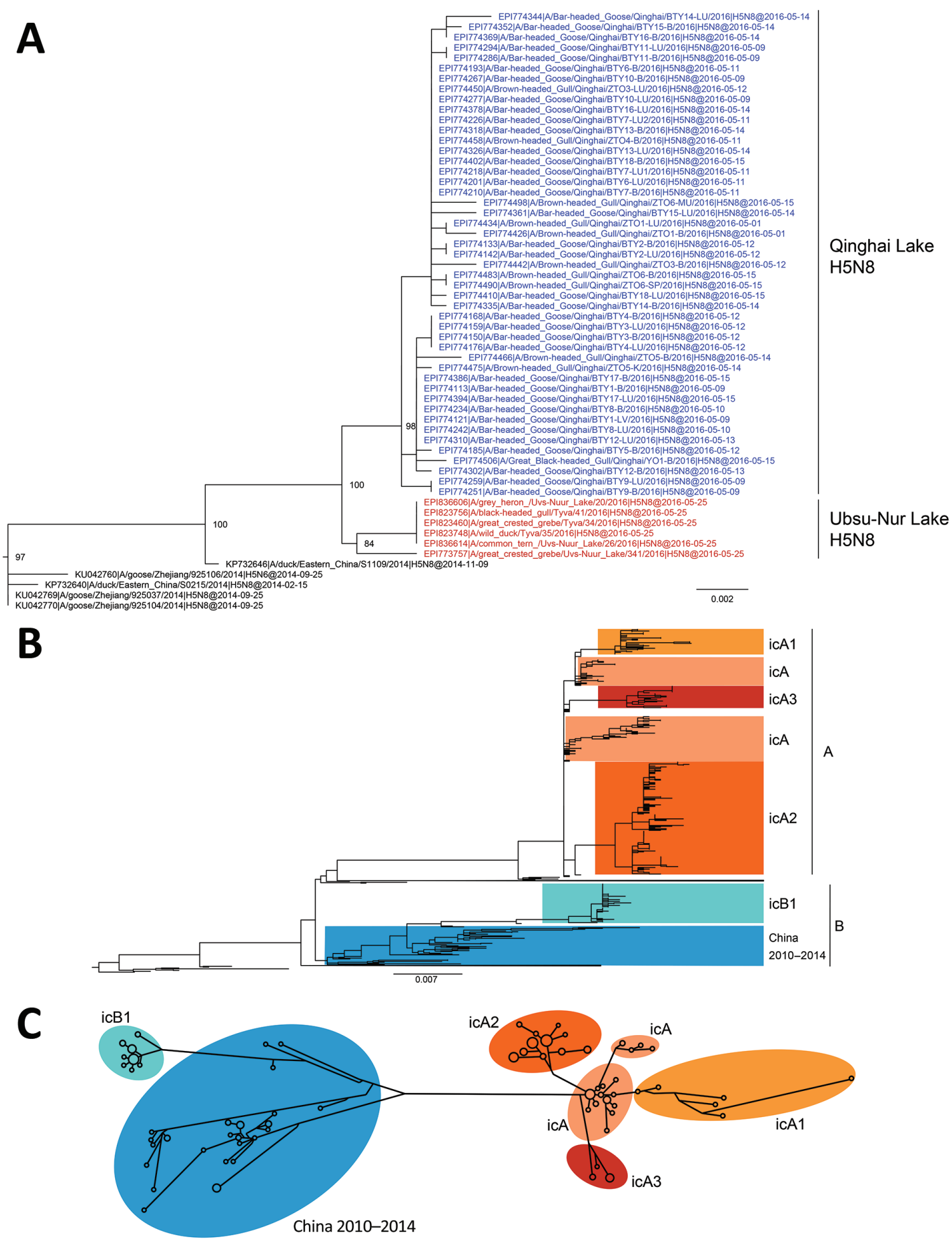

Figure 1. Phylogenetic analyses of 594 hemagglutinin (HA) sequences (1,704 nt) from clade 2.3.4.4 H5 influenza viruses. A) HA-coding sequence subtree from maximum-likelihood phylogenetic analysis of the clade 2.3.4.4 H5 viruses. Colored nodes: blue, Qinghai Lake H5N8 strains (this study); red, Ubsu-Nur Lake H5N8 strains. B) Maximum-likelihood phylogenetic tree of the clade 2.3.4.4 HA-coding sequences, rooted with A/Goose/Guangdong/1/96(H5N1). Scale bars indicate nucleotide substitutions per site. C) Median-joining phylogenetic network of the HA-coding gene sequences, including the most parsimonious trees linking the sequences. To simplify the network, nodes with only one sequence are not shown. Network branch lengths are proportional to the numbers of mutations. icA, intercontinental group $A$; icA1, intercontinental subgroup A1; icA2, intercontinental subgroup A2; icA3, intercontinental subgroup A3; icB1, intracontinental subgroup B1. 
internal genes share high nucleotide identity with those of the low pathogenicity avian influenza (LPAI) viral pool in waterfowl from Mongolia and other regions (online Technical Appendix Table 1).

Phylogenetic analysis confirmed that the 8 segments had different origins. In the HA, NA, and NS phylogenetic trees, the QH-H5N8 virus clustered with $\mathrm{H} 5 \mathrm{~N} 8$ viruses isolated in late May 2016 from wild waterfowl at Ubsu-Nur Lake (UN-H5N8), forming a monophyletic cluster (Figure 1, panel A; online Technical Appendix Figure 2, panels D, F, H). Unlike the H5N8 strains previously described in South Korea in 2014-2015 (Buan2-like, group A), this cluster fell within group B (Gochang1-like) H5N8 viruses, forming a novel subgroup, intracontinental group B (icB1) (Figure 1, panel B; online Technical Appendix Figure 2, panel D). Neighbor-joining phylogenetic network analysis of the HA segment of the clade 2.3.4.4 H5 viruses also supported the finding that the QH-H5N8 and UN-H5N8 strains form a monophyletic cluster and appear to have evolved independently from group A H5N8 viruses (Figure 1, panel C).

Phylogenetic trees constructed by using sequences from the internal genes (all but polymerase basic 1 [PB1]) show that $\mathrm{QH}-\mathrm{H} 5 \mathrm{~N} 8$ and $\mathrm{UN}-\mathrm{H} 5 \mathrm{~N} 8$ viruses are closely related to various LPAI viruses circulating in aquatic birds in Mongolia in 2015. The PB1 gene, however, originated from various LPAI viruses dispersed across a relatively large geographic region (East and South Asia) over a long period (2010-2015) (online Technical Appendix Figure 2).

We used molecular dating to estimate the timing of the reassortment events that led to the emergence of $\mathrm{QH}-$ H5N8 (online Technical Appendix Figure 3). The HA, NA, and NS genes were transferred from domestic waterfowl in eastern China to wild migratory birds in approximately October 2015, January 2016, and December 2015, respectively (Figure 2; online Technical Appendix Table 2). Other internal gene segments (except PB1) originated from Mongolian waterfowl during July 2014-January 2016 (Figure 2; online Technical Appendix Table 2, Figure 3). The PB1 segment differs from the other segments, and was transferred from a LPAI virus circulating among waterfowl in Asia in February 2014 (Figure 2; online Technical Appendix Table 2, Figure 3). Thus, the generation of QH-H5N8 in wild migratory birds appears to have been a complex process and was likely completed in early 2016 (Figure 2).

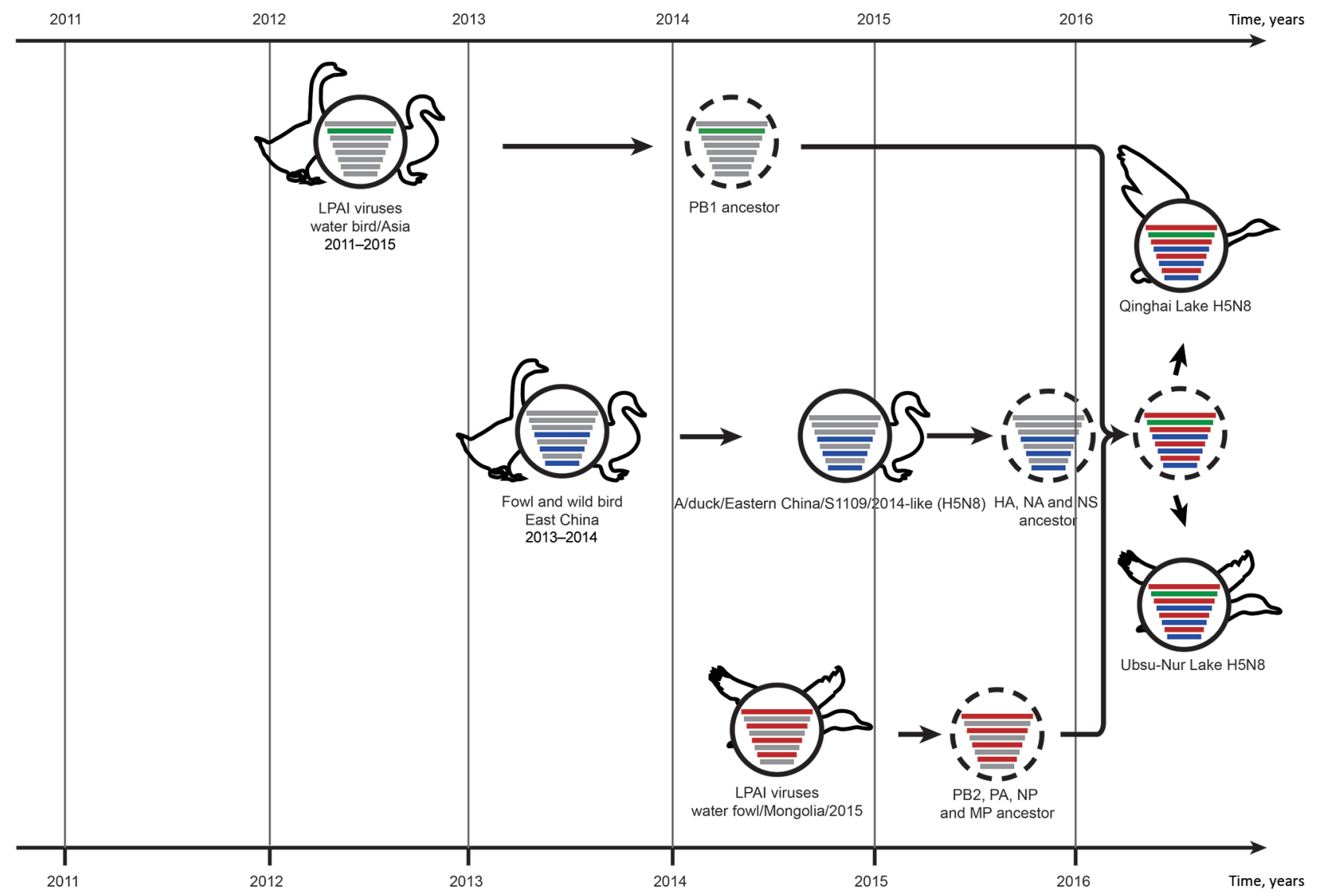

Figure 2. Hypothetical evolutionary pathway of influenza (H5N8) viruses from Qinghai Lake, China. Gene segments are colored according to their origins. Dashed virions indicate unidentified viruses. HA, hemagglutinin; LPAI, low pathogenicity avian influenza; MP, matrix protein; NA, neuraminidase; NP, nucleoprotein; NS, nonstructural protein; PA, acidic polymerase; PB, polymerase basic. 


\section{Conclusions}

We show that a group B H5N8 virus emerged in Qinghai Lake, China, causing deaths in wild migratory birds. Phylogenetic analysis indicates that the QH-H5N8 virus is the descendant of an unidentified triple-reassortant strain (Figure 2). The reassortment event may have occurred in waterfowl, and can be traced back to early 2016 . However, we cannot infer the geographic region where the reassortant virus was generated, because the gene constellation of the virus originated from different locations.

The absence of domestic poultry in the vicinity of Qinghai Lake strongly suggests that the virus was introduced to the area by wild birds. The deaths in Qinghai Lake occurred during May-June 2016, which corresponds with the breeding season for the affected species. In late May 2016, similar H5N8 strains were detected in wild migratory birds at Ubsu-Nur Lake, 1,600 km north of Qinghai Lake $(9,10)$. This finding suggests that the early summer movement of wild migratory birds from unknown southern sites to northern breeding grounds resulted in the introduction of $\mathrm{H} 5 \mathrm{~N} 8$ to Qinghai Lake and to Ubsu-Nur Lake, infecting a diverse population of breeding waterbirds.

Currently, we know of 3 HPAI H5N1 virus clades that have been introduced to wild migratory birds in Qinghai Lake, which is located near multiple migratory flyways: clade 2.2 in $2005(11,12)$, clade 2.3 .2 in $2009(13,14)$, and clade 2.3.2.1c in 2015 (15). On all 3 occasions, similar viruses were subsequently detected in other regions. Therefore, when wild birds left the breeding location for their wintering sites in the autumn of 2016, H5N8 virus could potentially have spread to other regions along the flyway. HPAI H5N8 viruses have already caused fatalities among wild birds or poultry in South Asia, Europe, the Middle East, and Africa (http://www.oie.int/) since late October 2016. Available genetic information shows that H5N8 strains isolated in other countries are highly similar to the QH-H5N8-like virus, suggesting that the QH-H5N8-like viruses may have already disseminated to other areas along the migratory flyways.

\section{Acknowledgment}

We thank the data submitters from the GISAID for their H5N8 sequences.

This study was supported by grants from the Special Project of Ministry of Science and Technology (2013FY113500), National Natural Science Foundation of China (31570026), intramural special grant for influenza virus research from the Chinese Academy of Sciences (KJZD-EW-L09 and KJZD-EW-L15), the Ministry of Science and Technology of China 973 Project (2015CB910501), and the Sanming Project of Medicine in Shenzhen (ZDSYS201504301534057). G.F.G. is a principal investigator in the National Natural Science Foundation of China
Innovative Research Group (81321063). W.S. was supported by the "Taishan Scholar" project of Shandong Province. Y.B. is supported by the Youth Innovation Promotion Association of Chinese Academy of Sciences. G.W. is the recipient of a Banting Postdoctoral Fellowship from the Canadian Institutes of Health Research and the President's International Fellowship Initiative from the Chinese Academy of Sciences.

Mr. Li is a postgraduate student at CAS Key Laboratory of Special Pathogens and Biosafety of Chinese Academy of Sciences. His primary research interest is the epidemiology of influenza viruses.

\section{References}

1. World Health Organization/World Organisation for Animal Health/ Food and Agriculture Organization (WHO/OIE/FAO) H5N1 Evolution Working Group. Revised and updated nomenclature for highly pathogenic avian influenza A (H5N1) viruses. Influenza Other Respi Viruses. 2014;8:384-8. http://dx.doi.org/10.1111/ irv. 12230

2. Zhao K, Gu M, Zhong L, Duan Z, Zhang Y, Zhu Y, et al. Characterization of three H5N5 and one H5N8 highly pathogenic avian influenza viruses in China. Vet Microbiol. 2013;163:351-7. http://dx.doi.org/10.1016/j.vetmic.2012.12.025

3. Lee YJ, Kang HM, Lee EK, Song BM, Jeong J, Kwon YK, et al. Novel reassortant influenza A(H5N8) viruses, South Korea, 2014. Emerg Infect Dis. 2014;20:1087-9. http://dx.doi.org/10.3201/ eid2006.140233

4. Wu H, Peng X, Xu L, Jin C, Cheng L, Lu X, et al. Novel reassortant influenza $\mathrm{A}(\mathrm{H} 5 \mathrm{~N} 8)$ viruses in domestic ducks, eastern China. Emerg Infect Dis. 2014;20:1315-8. http://dx.doi.org/10.3201/eid2008.140339

5. World Organisation For Animal Health. Updated on highly pathogenic avian influenza in animals (type H5 and H7), 20142015. Paris: The Organisation; 2015.

6. Lee DH, Torchetti MK, Winker K, Ip HS, Song CS, Swayne DE. Intercontinental spread of Asian-origin $\mathrm{H} 5 \mathrm{~N} 8$ to North America through Beringia by migratory birds. J Virol. 2015;89:6521-4. http://dx.doi.org/10.1128/JVI.00728-15

7. Verhagen JH, Herfst S, Fouchier RAM. How a virus travels the world. Science. 2015;347:616-7. http://dx.doi.org/10.1126/ science.aaa6724

8. Global Consortium for H5N8 and Related Influenza Viruses. Role for migratory wild birds in the global spread of avian influenza H5N8. Science. 2016;354:213-7. http://dx.doi.org/10.1126/science. aaf8852

9. Sims L, Khomenko S, Kamata A, Belot G, Bastard J, Palamara E, et al. H5N8 highly pathogenic avian influenza (HPAI) of clade 2.3.4.4 detected through surveillance of wild migratory birds in the Tyva Republic, the Russian Federation - potential for international spread. Food and Agriculture Organization. 2016;35 [cited 2016 Dec 7]. http://www.fao.org/3/a-i6113e.pdf

10. Lee DH, Sharshov K, Swayne DE, Kurskaya O, Sobolev I, Kabilov M, et al. Novel reassortant clade 2.3.4.4 avian influenza $\mathrm{A}(\mathrm{H} 5 \mathrm{~N} 8)$ virus in wild aquatic birds, Russia, 2016. Emerg Infect Dis. 2017 Feb [Epub ahead of print]. http://dx.doi.org/10.3201/ eid2302.161252

11. Liu J, Xiao H, Lei F, Zhu Q, Qin K, Zhang XW, et al. Highly pathogenic $\mathrm{H} 5 \mathrm{~N} 1$ influenza virus infection in migratory birds. Science. 2005;309:1206. http://dx.doi.org/10.1126/science.1115273

12. Wang G, Zhan D, Li L, Lei F, Liu B, Liu D, et al. H5N1 avian influenza re-emergence of Lake Qinghai: phylogenetic and 
antigenic analyses of the newly isolated viruses and roles of migratory birds in virus circulation. J Gen Virol. 2008;89:697-702. http://dx.doi.org/10.1099/vir.0.83419-0

13. Li Y, Liu L, Zhang Y, Duan Z, Tian G, Zeng X, et al. New avian influenza virus (H5N1) in wild birds, Qinghai, China. Emerg Infect Dis. 2011;17:265-7. http://dx.doi.org/10.3201/eid1702.100732

14. Hu X, Liu D, Wang M, Yang L, Wang M, Zhu Q, et al. Clade 2.3.2 avian influenza virus (H5N1), Qinghai Lake region, China, 20092010. Emerg Infect Dis. 2011;17:560-2. http://dx.doi.org/10.3201/ eid1703.100948
15. Bi Y, Chen J, Zhang Z, Li M, Cai T, Sharshov K, et al. Highly pathogenic avian influenza H5N1 Clade 2.3.2.1 c virus in migratory birds, 2014-2015. Virol Sin. 2016;31:300-5. http://dx.doi.org/10.1007/s12250-016-3750-4

Address for correspondence: Jianjun Chen, CAS Key Laboratory of Special Pathogens and Biosafety, Wuhan Institute of Virology, Chinese Academy of Sciences, Xiaohongshan 44\# Wuhan 430071, China; email: chenjj@wh.iov.cn

\section{December 2011: Zoonotic Infections}

- Risk for Rabies Importation from North Africa

- Worldwide Occurrence and Impact of Human Trichinellosis, 1986-2009

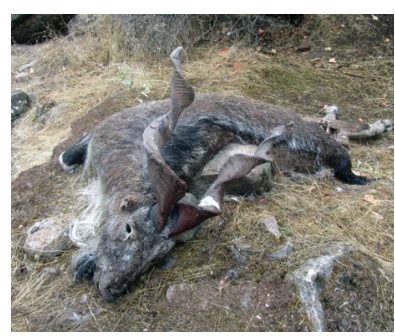

- Sealpox Virus in Marine Mammal Rehabilitation Facilities, North America, 2007-2009

- Transmission of Guanarito and Pirital Viruses among Wild Rodents, Venezuela

- Hepatitis E Virus in Rats, Los Angeles, California

- Enterovirus Co-infections and Onychomadesis after Hand, Foot, and Mouth Disease, Spain

- Experimental Infection of Horses with Hendra Virus/ Australia/Horse/2008/ Redlands

- Lineage and Virulence of Streptococcus suis Serotype 2 Isolates from North America

- West Nile Virus Infection of Birds, Mexico

- Isolation of Prion with BSE Properties from Farmed Goat
- Candidate Cell Substrates, Vaccine Production, and Transmissible Spongiform Encephalopathies

- Molecular Epidemiology of Rift Valley Fever Virus

- Novel Multiplexed HIV/ Simian Immunodeficiency Virus Antibody Detection Assay

- Astroviruses in Rabbits

- Host Genetic Variants and Influenza-Associated Mortality among Children and Young Adults

- Severe Human Bocavirus Infection, Germany

- Continuing Threat of Influenza (H5N1) Virus Circulation in Egypt

- Hepatitis E Virus Antibodies in Blood Donors, France

- Human Cardioviruses, Meningitis, and Sudden Infant Death Syndrome in Children

- Seroprevalence of Alkhurma and Other Hemorrhagic Fever Viruses, Saudi Arabia

- Knowledge of Avian Influenza (H5N1) among Poultry Workers, Hong Kong, China

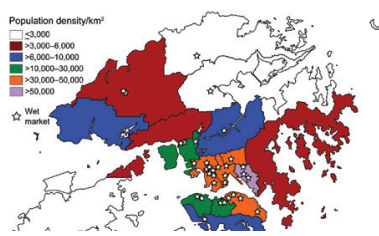

- Risk for Human African Trypanosomiasis, Central Africa, 2000-2009

- Animal Diseases Caused by Orbiviruses, Algeria

- Genogroup I and II Picobirnaviruses in Respiratory Tracts of Pigs

- Human Liver Infection by Amphimerus spp. Flukes, Ecuador

- Aedes aegypti Mosquitoes Imported into the Netherlands, 2010

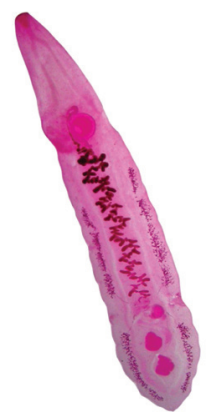

- Fatal Outbreak of Mycoplasma capricolum Pneumonia in Endangered Markhors

- African Swine Fever Virus Caucasus Isolate in European Wild Boars

- Novel Sylvatic Rabies Virus Variant in Endangered Golden Palm Civet, Sri Lanka

- Rickettsia parkeri in Amblyomma maculatum Ticks, North Carolina, 2009-2010

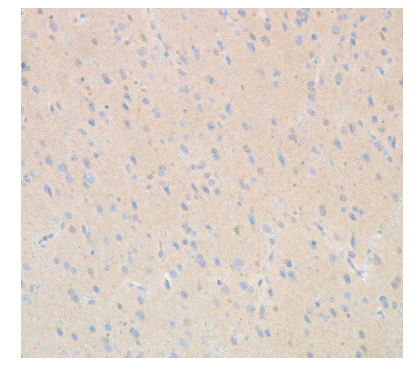

- Japanese Encephalitis Virus Genotype Replacement, Taiwan, 2009-2010

- Altitude-dependent Bartonella quintana Genotype C in Head Lice, Ethiopia

- Proximity to Goat Farms and Coxiella burnetii Seroprevalence among Pregnant Women

- Q Fever in Woolsorters, Belgium

- Coxiella burnetii Infection in Roe Deer during Q Fever Epidemic, the Netherlands

- Ranavirosis in Invasive Bullfrogs, Belgium

- Rift Valley and West Nile Virus Antibodies in Camels, North Africa

- Brucellosis, Taiwan, 2011

- Hemoptysis Associated with Leptospirosis Acquired in Hawaii

- Salmonella enterica in Pinnipeds, Chile

- Changing Perception of Avian Influenza Risk, Hong Kong, 2006-2010 